\title{
Autophagy maintains stemness by preventing senescence
}

Laura García-Prat*, Marta Martínez-Vicente, Eusebio Perdiguero, Laura Ortet, Javier Rodríguez-Ubreva, Elena Rebollo, Vanessa Ruiz-Bonilla, Susana Gutarra, Esteban Ballestar, Antonio L. Serrano, Marco Sandri \& Pura Muñoz-Cánoves*

\section{PAPER ABSTRACT}

During ageing, muscle stem-cell regenerative function declines. At advanced geriatric age, this decline is maximal owing to transition from a normal quiescence into an irreversible senescence state. How satellite cells maintain quiescence and avoid senescence until advanced age remains unknown. Here we report that basal autophagy is essential to maintain the stemcell quiescent state in mice. Failure of autophagy in physiologically aged satellite cells or genetic impairment of autophagy in young cells causes entry into senescence by loss of proteostasis, increased mitochondrial dysfunction and oxidative stress, resulting in a decline in the function and number of satellite cells. Re-establishment of autophagy reverses senescence and restores regenerative functions in geriatric satellite cells. As autophagy also declines in human geriatric satellite cells, our findings reveal autophagy to be a decisive stem-cell-fate regulator, with implications for fostering muscle regeneration in sarcopenia.

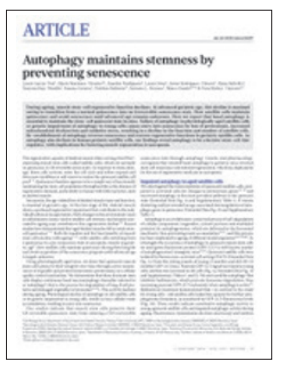

Citation: Nature 529, 37-42 (2016)

doi: $10.1038 /$ nature 16187

- RECEIVED 21 November 2014

- ACCEPTED 29 October 2015

- PUBLISHED ONLINE 6 January 2016

\section{SUMMARY}

Autophagy maintains youthfulness and rejuvenates aged stem cells. Muscle stem cells normally exist in a reversible nondividing state called quiescence. Our study shows that stemcell fitness during quiescence is maintained by autophagy, the regulated disassembly of cell components. Old stem cells lose autophagic capacity, provoking an irreversible senescent state. Reactivation of autophagy prevents senescence and rejuvenates aged stem cells.

\section{The problem}

In tissues with little turnover, stem cells (which repair tissues and organs) remain in a dormant, quiescent state. The stem cells of skeletal muscle (also called satellite cells) were thought to be quiescent most of the time, only activating in response to damage or stress ${ }^{1,2}$. Little was known about how these long-lived cells maintain quiescence or about their capacity to activate and repair damaged muscle. We hypothesized that satellite cells would progressively accumulate intracellular toxic debris over their lifespan, and would need an efficient 'clean-up' system to maintain protein homeostasis (proteostasis) ${ }^{3}$. These ideas led us to investigate the possible role of autophagy, a system used by cells to degrade damaged proteins and organelles in vesicular structures called lysosomes ${ }^{4,5}$. Recent evidence shows that satellite cells lose the capacity to maintain quiescence as they age ${ }^{6-9}$. Moreover, at advanced, geriatric life stages, satellite cells enter an irreversible state of proliferative block called senescence, leading to defective muscle regeneration ${ }^{8}$. So a second question that arose was what causes this quiescence-to-senescence transition during old age.

\section{The solution}

We found that in adult mice, satellite cells, despite their dormant state, maintain a basal autophagic activity. In old age, however, this activity is lost, causing the accumulation of damaged mitochondria. Mitochondria are the organelles of energy production, and the accumulated dysfunctional mitochondria generate high levels of reactive oxygen species (ROS), causing further damage to proteins and DNA. This loss of proteostasis provokes entry into senescence and impairs satellite-cell function. By reactivating autophagy or inhibiting ROS production in aged satellite cells, we were able to prevent senescence, restore stem-cell self-renewal, and reboot the capacity to form new muscle fibres. To confirm that dysfunctional autophagy underlies entry to senescence, we generated genetically modified mice in which autophagy is blocked in young satellite cells. These cells rapidly transit from quiescence to senescence and lose their regenerative potential. Forced loss of autophagy in young satellite cells thus causes premature ageing and disrupts stem-cell functions. 
The implications

The identification of autophagy as an obligatory activity for maintaining stem-cell fitness, despite these cells' low metabolic activity, not only advances the understanding of stem-cell biology but also suggests research avenues into regenerative-medicine applications. Although exactly how autophagy balances quiescence and senescence in muscle stem cells remains unknown, our studies demonstrate that it is a decisive factor in the switch between these two cell fates. It will be interesting to see if autophagy-inducing strategies based on dietary supplements and lifestyle changes can rejuvenate endogenous stem cells in old tissues and organs, and enhance regeneration. These strategies may also improve stem-cell engraftment in cell-based therapies for severe degenerative diseases. We hope that our findings will inspire other researchers to address these questions; their answers will illuminate new strategies for healthy ageing and combating ageing-associated diseases.

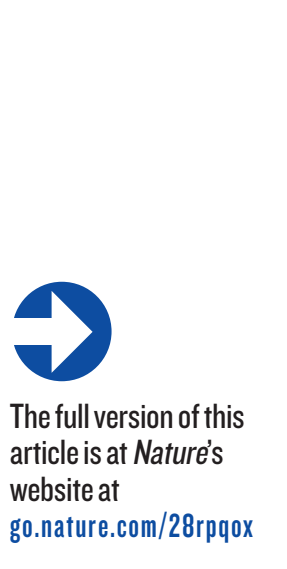

\section{GRAPHICAL ABSTRACT}

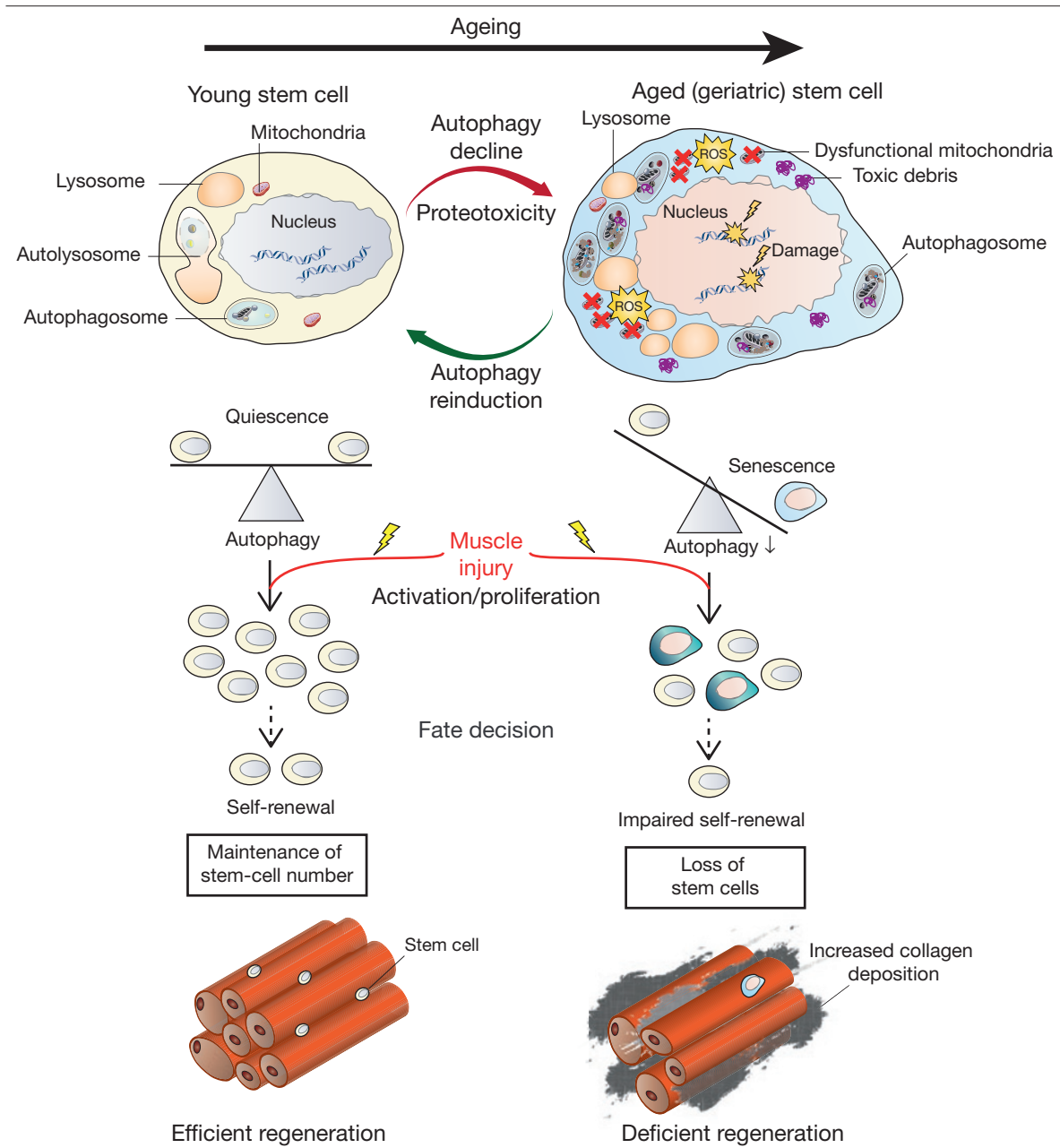

Proposed model of how age-impaired autophagy leads to muscle stem-cell senescence and regenerative decline. Muscle stem cells in young mice are in a dormant, quiescent state, and maintain their fitness through constitutive baseline autophagy. This autophagic activity functions as a homeostatic 'clean-up' mechanism that ensures intracellular proteostasis. With ageing, autophagic activity progressively declines, resulting in the accumulation of toxic cellular waste, including damaged proteins and mitochondria. The ensuing mitochondrial dysfunction in turn produces elevated levels of ROS and DNA damage. The proteotoxic stress in old stem cells (particularly at advanced, geriatric age) causes de-repression of the $16^{\text {INK4a }}$ locus and entry into a senescence-like state, instead of the functional quiescent state. After muscle injury, the senescent muscle stem cells in old mice cannot regenerate myofibres or repopulate the satellite-cell population by self-renewal. This senescent phenotype can be reversed by re-inducing autophagy pharmacologically or genetically, indicating that constitutive autophagy is required to maintain the muscle stem-cell pool and to preserve its regenerative potential.

\section{FURTHER READING}

1. Dumont, N. A., Wang, Y. X. \& Rudnicki, M. A. Intrinsic and extrinsic mechanisms regulating satellite cell function. Development $\mathbf{1 4 2}$ 1572-1581 (2015)

Review of intrinsic and extrinsic factors that regulate satellite-cell behaviour.

2. Brack, A. S. \& Rando, T. A. Tissue-specific stem cells: lessons from the skeletal muscle satellite cell. Cell Stem Cell 10, 504-514 (2012). This reviews the properties that satellite cells share with other stem-cell populations.

3. Kaushik, S. \& Cuervo, A. M. Proteostasis and aging. Nature Med. 21, 1406-1415 (2015). Review of proteostasis networks.

4. Cuervo, A. M. et al. Autophagy and aging: the importance of maintaining "clean" cells. Autophagy 1, 131-140 (2005). Review of molecular defects leading to the malfunctioning of two forms of autophagy.

5. Klionsky, D. J. et al. Guidelines for the use and interpretation of assays for monitoring autophagy (3rd edition). Autophagy 12, 1-222 (2016).

Guidelines for methods to examine macroautophagy and related processes.

6. Chakkalakal, J. V. et al. The aged niche disrupts muscle stem cell quiescence. Nature 490, 355-360 (2012).

An age-dependent change that influences muscle stem-cell quiescence and function.

7. Sousa-Victor, P. et al. Muscle stem cell aging: regulation and rejuvenation. Trends Endocrinol. Metab. 26, 287-296 (2015).

This article reviews muscle stem-cell biology, focusing on rejuvenation of old satellite cells.

8. Sousa-Victor, P. et al. Geriatric muscle stem cells switch reversible quiescence into senescence. Nature 506, 316-321 (2014) This shows that resting muscle stem cells switch to an irreversible senescence state.

9. Almada, A. E. \& Wagers, A. J. Molecular circuitry of stem cell fate in skeletal muscle regeneration, ageing and disease. Nature Rev. Mol. Cell Biol. 17, 267-279 (2016). Review of commonalities in molecular mechanisms regulating satellite-cell fate.

\section{RELATED CONTENT}

\author{
Autophagy rescues muscle ageing \\ P. Strzyz \\ go.nature.com/28twyuk
}

Autophagy is a key factor in maintaining the regenerative capacity of muscle stem cells by promoting quiescence and preventing senescence

X. Wen \& D. J. Klionsky

go.nature.com/28twxj6 